\title{
FUNDAMENTOS ECONÓMICOS, FALLOS DE MERCADO Y EXPORTACIÓN DE CAPITALES: AMÉRICA LATINA Y EL MERCADO DE LONDRES, 1880-1913
}

\author{
ECONOMIC FUNDAMENTALS, MARKET FAILURES \\ AND CAPITAL EXPORTS: LATIN AMERICA AND \\ LONDON'S CAPITAL MARKET, 1880-1913
}

\author{
Juan H. Flores* \\ Université de Genève, Ginebra, Suiza, <juan.flores@unige.ch>
}

\begin{abstract}
Resumen. El presente trabajo analiza los mecanismos mediante los cuales la estructura del mercado de emisión de deuda soberana en Londres entre 1880 y 1913 afectaba los costos de los empréstitos de los gobiernos de países prestatarios. Si bien la reciente literatura ha debatido sobre la importancia de los fundamentos económicos en la percepción de riesgo país de los inversionistas, este trabajo enfatiza que su papel es secundario bajo ciertas circunstancias, y que la naturaleza de dicha percepción es dinámica. Mi demostración introduce el concepto de "relación bancaria" para describir la relación entre la banca internacional y los países prestatarios e ilustro su importancia para explicar la crisis Baring de 1890.
\end{abstract}

Palabras clave: deuda soberana, banca comercial, fallos de mercado, globalización.

Abstract. This paper analyzes the mechanisms through which the structure of the market for sovereign debt in London affected borrowing costs in the period of 1880-1913. The traditional literature has emphasized the role of the economic fundamentals to explain the evolution of sovereign risk. More recent works, however, have demonstrated that economic fundamentals may be irrelevant under certain conditions, and that their effect on risk premia is dynamic in time. I use the concept of "relationship banking" to describe the relations between financial intermediaries, borrowing governments and borrowing costs, and provide a new explanation of the Baring crisis of 1890 .

Key words: sovereign debt, investment banking, market failures, globalization.

Fecha de recepción: mayo de 2011. Fecha de aceptación: octubre de 2011.

* Agradezco los comentarios de los tres dictaminadores anónimos, y el apoyo financiero del Fondo Nacional de Investigación Suizo.

Am. Lat. Hist. Econ., año 19, núm. 3, septiembre-diciembre, 2012, pp. 5-30 
Las experiencias de una generación son generalmente inútiles para la siguiente: y es por eso que los eventos históricos invocados como elementos de demostración no sabrán servir. La única utilidad es de demostrar hasta qué punto las experiencias tienen que repetirse una y otra vez para ejercer alguna influencia, y ayudar a eliminar un error sólidamente implantado. ${ }^{1}$

\section{INTRODUCCIÓN}

$\mathrm{E}$ 1 presente ensayo expone algunos de los principales resultados recientes de trabajos sobre la historia económica y financiera del periodo de la llamada "primera globalización", particularmente sobre los aspectos relacionados con el análisis microeconómico de las relaciones financieras internacionales. ${ }^{2}$ Hacemos un especial énfasis en los incentivos de la parte de los gobiernos prestatarios para adoptar políticas compatibles con la "disciplina de mercado", y explicaremos cómo influyen las instituciones financieras en la percepción de riesgo por parte de los inversionistas (riesgo soberano). ${ }^{3}$

Una perspectiva de largo plazo nos ofrece un enfoque especialmente enriquecedor sobre la trayectoria de lo que se denomina "políticas económicas adecuadas" a lo largo del tiempo. En términos generales, podemos verificar que la teoría económica ha experimentado un proceso basado en lo que Thomas Kuhn denominó cambio de paradigma, según el cual, el progreso científico partiría de cambios abruptos de paradigmas o conceptualizaciones específicas a cada periodo. En este sentido, una teoría dominante tendería a ser remplazada por una nueva en el momento en que la primera encontrase dificultades experimentales que pusiesen en evidencia la incompatibilidad del paradigma dominante. Existen casos históricos en varios aspectos del pensamiento económico: el paradigma sobre el progreso natural según Adam Smith, Malthus o David Ricardo duró más de 100 años para ser desafiado por Friedrich List o Christian Rother en el siglo XIX, y más tarde por los economistas de desarrollo de la segunda mitad del siglo XX. Más recientemente, Landes ofrece una visión general sobre el cambio de paradigmas en la teoría sobre el crecimiento económico. ${ }^{4}$

\footnotetext{
${ }^{1}$ Bon, Psychologie, 1895.Traducción del francés de Juan H. Flores.

${ }^{2}$ Entre estos trabajos los que más destacamos son los de Eichengreen y Bordo, "Crises", 2002; Flandreau, "Crises", 2003; Flandreau y Flores, "Bonds", 2009, y "Bondholders", 2011.

${ }^{3}$ Un análisis reciente sobre este tema es Mosley, Global, 2003.

${ }^{4}$ Landes, "Why", 1990.
} 
Si nos concentramos en las finanzas internacionales, el debate sobre los beneficios de la libertad de los movimientos internacionales de capital tiene una larga historia. Ragnar Nurske había advertido sobre los riesgos inherentes del sistema liberal de la década de 1920, y J. M. Keynes y Harry Dexter White lo debatieron en el periodo posterior a la segunda guerra mundial, cuando la regulación de los mercados de capitales parecía imprescindible. ${ }^{5}$ Sin embargo, las virtudes potenciales de un sistema liberal al movimiento internacional de capitales nunca han sido negadas: la posibilidad de eliminar las restricciones impuestas por el ahorro interno, de incrementar la inversión privada y pública y, por ende, fomentar el crecimiento económico. ${ }^{6}$ Ahora bien, ante el riesgo de un aumento continuo en la volatilidad financiera, el debate se ha concentrado en la búsqueda de una arquitectura financiera internacional estable, y en encontrar una explicación coherente sobre el significado de las crisis financieras internacionales. ${ }^{7}$ En el fondo, se trata también de analizar la dirección y el volumen de los flujos internacionales de capital y la posible existencia de un eje conductor en estos movimientos.

Sobre esta última cuestión, el razonamiento sería el siguiente. Los capitales irían ahí donde la percepción rentabilidad-riesgo resulte más atractiva. Esta percepción determina el volumen de capital que cada país recibirá, y es función de ciertas variables que pueden ser, entre otras, macroeconómicas, institucionales, psicológicas o culturales. ${ }^{8}$ La dificultad radica en que dichas percepciones cambian en el tiempo, por lo que la comprensión de sus cambios y la identificación de las razones de la ascendencia de una percepción sobre otra previamente dominante resulta relevante para el estudio de la economía. De hecho, la crisis actual ha provocado una serie de cuestionamientos sobre el estado de la ciencia económica y su orientación metodológica. ${ }^{9}$ Por ejemplo, el debate en va-

\footnotetext{
${ }^{5}$ Nurske, "International", 1954. Sobre el debate histórico relacionado con los beneficios y los riesgos de los movimientos de capitales y sobre la historia de las crisis financieras véase Marichal, Nueva, 2010.

${ }^{6}$ Aquí cabría mencionar la notable excepción de Rodrik y Subramanian, quienes demuestran empíricamente que la restricción del ahorro ha sido menos importante, para muchos países en vías de desarrollo, que las condiciones institucionales y económicas para invertir. Su estudio se inscribe en la literatura sobre el impacto nulo del capital internacional sobre el crecimiento económico entre 1970 y 2004. Rodrik y Subramanian, "Why”, 2009.

${ }^{7}$ Véase la exposición de dicho debate en Eichengreen, Capital, 2003.

${ }^{8}$ Sobre las variables políticas véanse Flandreau, "Home", 2006, el debate sobre el impacto del financiamiento de Londres en las colonias inglesas en Accominotti, Flandreau y Rezzik, "Spread", 2011, y Ferguson y Schularick, "Empire", 2006. Las variables culturales se introducen como factor explicativo en Clemens y Williamson, "Wealth", 2004, y en los modelos gravitaciones del comercio internacional (que incluyen una variable sobre lengua común para explicar el volumen de comercio bilateral), cuyo comportamiento está muy ligado al de los mismos flujos de capital.

${ }^{9}$ Véase, por ejemplo un artículo reciente por DeLong, "Economics”, 2011.
} 
rios países sobre la necesidad de disminuir el déficit público incorpora argumentos tradicionales con elementos innovadores sobre la estructura de las economías, la integración internacional comercial y financiera y la regulación bancaria. Hoy en día, presenciamos cierta divergencia de criterios en cuanto a la política macroeconómica adecuada compatible con un crecimiento económico sostenido a largo plazo y las exigencias de los mercados financieros en el corto plazo, inclusive en países desarrollados como Estados Unidos o Inglaterra.

Nuestro trabajo analiza el cambio de percepción en los mercados de capitales internacionales después de la crisis de 1890, y argumenta que el cambio de paradigma estuvo acompañado de un cambio en la organización industrial del mercado de emisión de deuda pública. Este episodio se inscribe dentro del periodo de la llamada primera globalización financiera, entre 1870 y 1913. Intentaremos demostrar que las percepciones sobre "buenas políticas económicas" son dinámicas y que dependen de la rentabilidad esperada pero también de las pérdidas no explicadas por los modelos dominantes. La estructura de los mercados de capitales está muy ligada a este cambio de percepciones, puesto que los inversionistas pueden ejercer presión sobre los intermediarios financieros y su función como supervisor delegado si los resultados no son los esperados, y esto puede tener un impacto sobre el volumen de las inversiones internacionales.

Este trabajo está dividido en tres partes. En la primera, haré una breve revisión de las principales preguntas analizadas sobre el periodo entre 1880 y 1913, y justificaremos su relevancia para trazar paralelos con el periodo actual de liberalización financiera. En la segunda parte explico el funcionamiento de los mercados de capitales de hace más de 100 años, y sitúo este funcionamiento en un marco teórico contemporáneo. En la tercera parte mostraré que la crisis Baring de 1890 no puede ser explicada únicamente por el deterioro de los fundamentos económicos en Argentina, y que los fallos del mercado internacional de capitales tuvieron un papel al menos tan importante como la evolución económica en dicho país. La crisis, no obstante, produjo importantes cambios en la percepción de riesgo de ciertas políticas económicas. En efecto, la política fiscal relevó a la apertura comercial como una de las variables más importantes para explicar la evolución del riesgo soberano, y la producción de publicaciones y estudios económicos por parte de los intermediarios financieros y los inversionistas aumentó de manera importante. Concluimos con algunas especulaciones en lo concerniente a la estructura actual de los mercados de capitales y sobre el tipo de regulación que pudiera ser beneficiosa para el buen funcionamiento de dichos mercados. 
Am. Lat. Hist. Econ., año 19, núm. 3, septiembre-diciembre, 2012, pp. 5-30

\section{EL DEBATE Y LA IMPORTANCIA DEL PERIODO \\ DE LA "PRIMERA GLOBALIZACIÓN"}

Los eventos económicos y financieros de las décadas de 1980 y 1990 obligaron a los economistas a buscar nuevos elementos que les ayudasen a enfrentar los nuevos desafíos de las finanzas internacionales, a medida que la liberalización y la innovación financieras, así como los avances tecnológicos permitían, de manera creciente, el recurso al financiamiento por medio de los mercados bursátiles internacionales (arm's length) a un número creciente de países. ${ }^{10}$ Desde nuestro punto de vista, hemos podido verificar que las crisis mexicana y asiática hicieron caer ciertos paradigmas para ver emerger nuevos. Por ejemplo, las reformas en el sector financiero y la aparente estabilidad macroeconómica como bases para el crecimiento económico y para fomentar la inversión privada probaron ser insuficientes; la literatura posterior a estas crisis se enfocó en la naturaleza de los flujos de capital (los llamados "golondrinos") como fuente de inestabilidad financiera, así como el papel del déficit en cuenta corriente y la apreciación en el tipo de cambio real, consecuencia del régimen de tipo de cambio fijo utilizado para frenar la inflación. ${ }^{11}$ El debate en la literatura se concentró sobre temáticas que habían quedado de lado probablemente desde la crisis de la deuda de 1982 o, incluso, desde los estudios sobre los defaults de la década de 1930. Por lo mismo, nuestra comprensión sobre temas tales como contagio o riesgo moral era, y probablemente es, en el mejor de los casos, imprecisa. ${ }^{12}$ Aun mientras cientos de nuevos artículos han sido publicados desde entonces, la crisis actual ha puesto de manifiesto que nuestra comprensión de las consecuencias de la liberalización y desregulación financieras sigue siendo muy limitada.

A pesar de lo anterior, la incertidumbre ha sido evocada frecuentemente como fuente de inestabilidad y ha sido relacionada con modelos de asimetría de información, el papel de los intermediarios financieros y el problema de los distintos conflictos de interés en el sector financiero. De hecho, el término "asimetrías de información" es muy vasto y tradicional

\footnotetext{
${ }^{10}$ Es decir, por la vía de mercados anónimos, o en este caso, por la vía de la emisión de obligaciones en los mercados de capitales internacionales.

${ }^{11}$ Véase por ejemplo el artículo de Radelet y Sachs, "East", 1998.

${ }^{12}$ De hecho, la literatura sobre el tema no ofrece una definición única de "contagio". Algunos economistas como Rudiger Dornbusch admitieron que todavía queda mucho que aprender sobre este fenómeno. Dornbusch, Chul y Claessens, “Contagion”, 2000. Alan Greenspan explicaba más generalmente: "We do not as yet fully understand the new system's dynamics. We are learning fast, and need to update and modify our institutions and practices to reduce the risks inherent in the new regime. Meanwhile, we have to confront the current crisis with the institutions and techniques we have." Discurso frente al Senado de Estados Unidos sobre la crisis asiática el 3 de marzo de 1998.
} 
de la literatura económica convencional; es el mismo que aparece como una de las causas del origen mismo de los intermediarios financieros. Después de una serie de reformas regulatorias e institucionales, la arquitectura financiera internacional actual sigue sin resolver aquellos elementos que ocasionan volatilidad e incertidumbre en los mercados financieros, y los conflictos de interés parecen estar más presentes que nunca. Hace ya tiempo que el Fondo Monetario Internacional (FMI) ha sido acusado de ocasionar riesgo moral entre los inversionistas, y su existencia ha sido cuestionada por provocar los problemas que en el papel tendría que resolver. ${ }^{13} \mathrm{~A}$ su vez, las agencias de notación son señaladas por no poder prevenir a tiempo a los inversionistas sobre los riesgos en ciertos países o empresas. ${ }^{14}$ En general, los gobiernos acusan a los mercados de introducir inestabilidad a sus economías (sobre todo en periodos de aflicción financiera) y afectar así su desarrollo castigando el aparato productivo de sus países.

Así pues, el estado actual de la arquitectura financiera global impone un nuevo desafío a la teoría económica contemporánea. El proceso de aprendizaje en distintos ámbitos como el institucional, el regulatorio y el puramente económico presenta discontinuidades y ha sido ocasionalmente violento. La historia económica ofrece paralelos interesantes con periodos de alta innovación financiera, integración creciente de los mercados de capitales y crisis financieras. El final del siglo XIX y principios del xx emergió como un periodo particularmente propicio para el análisis de cuestiones de interés actual. ${ }^{15}$ En efecto, ciertos estudios muestran que la integración financiera más importante a escala histórica ha sido la de aquel periodo. ${ }^{16}$ Otros autores argumentan que la exportación de capita-

${ }^{13}$ Dreher, Axel, "Does the IMF Cause Moral Hazard? A Critical Review of the Evidence", 23 de febrero de 2004, Social Science Research Network, <http://ssrn.com/abstract=505782>. [Consulta: 24 de abril de 2011.] Los dos grandes problemas ligados con las asimetrías de información son el riesgo moral y la selección adversa. El primero tiene que ver con un comportamiento de la parte del agente que resulta distinto al pactado en la transacción económica. El segundo existe cuando el agente (o vendedor) no revela toda la información sobre el producto. Akerlof, "Market”, 1970, expone el trabajo pionero sobre dicha problemática.

${ }^{14}$ Ferri, Lui y Stiglitz, "Procyclical”, 1999.

${ }_{15}$ Todavía poco antes de la crisis que comenzó en 2007, al introducir los términos "Economic Financial History" en la base de datos de Econlit, más de 60\% de los 231 resultados entre 1995 y 2004 estaban relacionados con el periodo 1870 y 1913. Cuando se introducía el término "globalización financiera" entre 1995 y 2004, los tres estudios históricos de los 42 resultados utilizaban al periodo 1870-1913 como punto de comparación con el presente. La crisis actual ha propiciado cierto cambio de orientación hacia el periodo comprendido entre las dos guerras mundiales, aunque por distintas razones que están relacionadas con la expansión de la crisis.

${ }^{16}$ Véanse, por ejemplo, Quinn, "Capital”, 2003, y Flandreau y Zumer, Making, 2004. Bordo, Eichengreen e Irwin, "Globalization”, 1999, y Obstfeld y Taylor, "Globalization”, 2003, argumentan, por otro lado, que la integración financiera actual es más profunda y completa que en el periodo anterior a 1914 . 
les, como proporción del producto interno bruto (PIB), nunca ha sido tan elevada como a finales del siglo XIX. ${ }^{17}$

Esta época dorada del patrón oro y de la integración financiera internacional fue acompañada de un aumento generalizado del comercio internacional y del crecimiento económico, aunque también tuvo un lado oscuro puesto que no escapó a los accidentes ni a los eventos violentos de inestabilidad global. Episodios con fuertes crisis financieras internacionales incluyen la crisis de 1873 (crack bursátil, crisis bancarias y defaults de deuda soberana en varios países como el imperio Otomán o Perú), en 1890 (la crisis Baring con una caída generalizada en los movimientos internacionales de capital) o en 1907 (crisis bancarias en distintos países como Estados Unidos o Italia). Muy frecuentemente, estas crisis tuvieron el potencial para tener repercusiones aun más importantes, por lo que la intervención pública fue requerida en varios casos para limitar los estragos. Conceptos como "contagio" o "rescate financiero" ya existían en las mentes de los economistas. Sin embargo, a diferencia de nuestra época, la arquitectura financiera internacional de aquel entonces debía lidiar con los problemas sin la ayuda de instituciones supranacionales y apoyarse casi únicamente por el mercado mismo. Por el contrario, existieron organismos privados cuyo fin era la defensa de los intereses de los inversionistas (como el Corporation of Foreign Bondholders, inglés, o la Association de Détenteurs de Titres Étrangers, francesa, por mencionar los más importantes), y una regulación estricta sobre los prestatarios que buscaban acceso a las principales plazas financieras internacionales. El contexto pues, parece invitarnos a comprender mejor el mundo de hace más de 100 años tanto en sus fortalezas como en sus debilidades, y a entender que nuestro sistema internacional de capitales no ha sido el resultado natural de las fuerzas de mercado ni el puerto final de los cambios continuos desde el final de Bretton Woods.

\section{FunCiOnAmiENTO DE LOS MERCADOS DE CAPITALES, 1870-1913}

Toda exposición sobre el funcionamiento de los mercados de capitales a finales del siglo XIX desde una perspectiva contemporánea tendría que comenzar con un recuento general sobre el estado de la tecnología financiera y de las comunicaciones. Sobre el primero, cabe señalar que muchos de los instrumentos utilizados en aquel entonces se asemejan bastante a los contemporáneos (si bien menos sofisticados) y en todo caso, contamos con la evidencia suficiente para sostener que los análisis riesgo-rendimiento de

${ }^{17}$ Stone, Global, 1999, y Edelstein, "Foreign”, 1994. 
los inversionistas incorporaban muchos elementos presentes en la actualidad. ${ }^{18}$ Por otro lado, la evidencia sobre la tecnología disponible en periodos anteriores permite darnos una idea sobre los problemas relacionados con la obtención de información rápida y confiable con los que los inversionistas debían lidiar. Es necesario recordar que los sistemas de correo marítimos hicieron progresos enormes entre 1870 y 1913. En el caso de la ruta de Londres a Buenos Aires, se pasó de 27 días a menos de doce, tal como podemos comprobar en los reportes de la prensa, en periódicos tales como The Economist o The Times, ambos de Londres. No obstante, para los fines de nuestro análisis, la verdadera revolución de las comunicaciones provino de la instalación del telégrafo trasatlántico, que empezó a tener uso público desde 1858, conectando Europa con el norte del continente americano, y en 1874 se inauguró la conexión de Europa con América del Sur. A finales de la década de 1870, todo individuo a los dos lados del continente contaba con varias rutas telegráficas comerciales que ofrecían sus servicios a distintas tarifas. En cuanto al tiempo de transmisión, hacia 1913 un mensaje telegráfico tardaba menos de once minutos. De hecho, por el comportamiento de los precios de los títulos y obligaciones cuyas transacciones se llevaban a cabo en ambos lados del Atlántico, podemos decir que los mercados estaban completamente integrados. ${ }^{19}$

En cuanto al aspecto institucional, la regulación internacional del flujo de capitales fue prácticamente inexistente, y solamente en casos nacionales se trataron los riesgos y se debatieron las medidas a adoptar para evitar mayores crisis en sus sectores financieros. ${ }^{20} \mathrm{El}$ proceso de endeudamiento internacional tuvo lugar, en apariencia, en un contexto que se caracteriza por la ausencia de un "doctor monetario" -entiéndase FMI- o prestamista de ultima instancia internacional. Por lo mismo, los inversionistas o compradores de los títulos de deuda de gobiernos extranjeros tenían fuertes incentivos para adquirir la información sobre las condiciones económicas de aquellos países. ${ }^{21} \mathrm{~A}$ partir de publicaciones contemporáneas como

\footnotetext{
${ }^{18}$ Este tipo de paralelos se encuentran en el libro de Mauro, Sussman y Yafeh, Emerging, 2006, quienes proveen un análisis detallado de dichos instrumentos financieros.

${ }_{19}$ Se pueden encontrar pruebas estadísticas formales y una discusión más detallada sobre el estado de la tecnología en Ahvenainen, European, 2004; Flores, "Lorsque”, 2004, o Pike y Winseck, "Communication", 2007. 98-125.

${ }^{20}$ Este fue el caso de Inglaterra luego de la crisis de 1873. Véase Marichal, Century, 1989, pp.

${ }^{21}$ Durante este periodo no se contaban con medidas estándar de la actividad económica como el PIB o el producto nacional bruto (PNB), pero algunos estadísticos proveían estimaciones basadas en su concepto de riqueza. Véase por ejemplo, el diccionario de estadísticas económicas de Mulhall, Mulhall's, 2008. Para países como Argentina o Australia, se miraba la evolución en la cantidad de ganado y ovejas como aproximación de la actividad económica. Véase "The financial position of Chili and the Argentine Republic compared", South American Journal, 11 de septiembre de 1889.
} 
Fenn's Compendium of the English and Foreign Funds..., The Investors Monthly Manual o Statesman Yearbook podemos saber qué tipo de variables interesaban a los inversionistas, y conocer su percepción sobre las políticas económicas adecuadas de cara a la rentabilidad y el riesgo de los capitales que ingresaban en los países que acudían a Londres para financiarse. Una discusión detallada al respecto se encuentra en el Fenn's Compendium de 1883, donde se revisan distintos indicadores sobre la sostenibilidad de la deuda de un país tomando en cuenta la utilización de los fondos prestados y el estado de la actividad económica. En la década de 1880, el monto de la deuda era evidentemente una variable que los inversionistas veían con mucho interés. Otras variables de particular importancia eran la apertura comercial, el aumento de las exportaciones y en general el estado de la cuenta corriente. Hacia mediados de la década de 1890 estas variables pierden cierta relevancia a favor de variables relacionadas con el déficit fiscal y el servicio anual de la deuda pública, como lo muestran Flandreau y Zumer. ${ }^{22}$ La pregunta, por tanto, es bajo qué principios operó este cambio súbito de la variable relevante para determinar la prima de riesgo, y en qué medida el modelo económico dominante comenzó a desmoronarse. La gestación de la crisis de 1890 en Argentina ocurrió en un entorno de aumento generalizado del endeudamiento (tanto en el ámbito nacional como en el provincial y municipal) pero también de fuerte expansión económica sostenida por el aumento en el comercio internacional. No es de sorprenderse, por lo tanto, que las variables fiscales pasaran a ocupar un lugar prioritario en el análisis de riesgo país hecho por los inversionistas. Lo que extraña es más bien la confianza continua entre los inversionistas a pesar de los malos augurios abundantes en la prensa y en el deteriorado estado de la macroeconomía argentina.

Para entender este cambio súbito de comportamiento generalizado de la parte de los inversionistas, tendríamos que hacer una revisión microeconómica de los mercados de capitales y en particular el papel de los intermediarios financieros. A grandes rasgos, sabemos que los inversionistas delegaban las funciones de supervisión sobre los países prestatarios a los merchant bankers, encargados de las emisiones de estos títulos (función similar a la de muchos bancos de inversión hoy en día) y que tenían relación directa con los gobiernos implicados: se trataba de dar seguimiento al riesgo de impago de aquellos países. Gran parte de la historiografía sobre la época argumenta que las relaciones banco-gobierno eran únicas: existían frecuentemente monopolios o cuasimonopolios de los bancos sobre las emisiones de cada país. ${ }^{23}$ Sin embargo, esto no era siempre el caso.

\footnotetext{
${ }^{22}$ Flandreau y Zumer, "Making”, 2004.

${ }^{23}$ Véase por ejemplo Cairncross, Home, 1953.
} 
Algunos países dejaban actuar la competencia entre los bancos para obtener mejores términos y menores costos sobre los empréstitos. Era particularmente el caso de países bien establecidos, como Francia (antes de que concentrara sus emisiones en París) o Rusia. Curiosamente, esto también era el caso de otros menos establecidos o con un historial negativo como pagadores, como por ejemplo Turquía o México, quienes cambiaban de banco emisor continuamente. ${ }^{24}$ Estas relaciones eran importantes para la percepción de riesgo de los bonos de cada país, por dos razones principales: una, por la cantidad de información producida por un banco gracias a sus relaciones con los países con quienes trataba, y dos, porque los bancos tenían distinta reputación en cuanto a la rentabilidad y el riesgo de los productos financieros que traían al mercado. ${ }^{25}$ Resulta por tanto relevante hacer una revisión breve del papel de los bancos en el mecanismo de emisión de deuda y exponer los motivos de las ventajas de las relaciones de largo plazo entre un banco y su prestatario.

Podemos distinguir cuatro etapas en el mecanismo de emisión de deuda del siglo XIX en las principales plazas financieras: 1) la búsqueda de fondos; 2) la elección del sistema de emisión; 3) la preparación de la emisión; 4) la colocación de los títulos. El mecanismo se define en las primeras dos etapas, y el documento donde se establece este mecanismo, así como las condiciones y costos es el contrato de deuda. ${ }^{26}$

\section{La búsqueda de fondos}

Los gobiernos eran por lo general los primeros agentes en el proceso cuando comenzaban la búsqueda de fondos. En algunos países, los gobiernos votaban "leyes" que permitían al poder ejecutivo la emisión dentro o fuera del país de títulos de deuda para algún proyecto (entiéndase infraestructura, medios de comunicación o de transporte, gastos de guerra). Para muchos de los países denominados "nuevos", los gobiernos nombraban un agente para negociar un empréstito con los banqueros europeos. Sin embargo, también podía presentarse el caso contrario, en el que los bancos, por medio de casas comerciales u otros agentes, podían proponer sus servicios a los gobiernos.

\footnotetext{
${ }^{24}$ Los libros de Ludlow y Marichal, Siglo, 1998, y Marichal, Century, 1989, hacen excelentes descripciones sobre la historia de la deuda en México y en América Latina.

${ }^{25}$ En particular, Flandreau y Flores han demostrado que los bancos de alta reputación, Rothschild y Baring, ofrecían títulos de deuda ex-ante menos riesgosos, y ex-post más rentables. Véase Flandreau y Flores, "Bondholders", 2011.

${ }^{26}$ Véase una discusión más detallada en Flores, “Competition”, 2010.
} 


\section{La elección del sistema de emisión}

Existían varias posibilidades que de hecho se definían desde la primera etapa. La primera posibilidad era el sistema de "venta a comisión": los bancos se limitaban a tomar las suscripciones para la compra de títulos de la parte de los inversionistas y a realizar todos los trámites necesarios para la emisión de los títulos. Los bancos demandaban comisiones por cada servicio. Otro sistema era el de la formación de sindicatos de bancos (los sindicatos podían, sin embargo, realizar las "ventas a comisión", aunque en realidad, uno de los objetivos de los bancos era la garantía de la colocación de los títulos). En París, podían existir dos tipos de sindicatos: los sindicatos de adquisición en "firme" y los sindicatos de "garantía". De acuerdo con el primer tipo de sindicato, los bancos suscribían o compraban en firme una cierta cantidad de títulos. Dentro del segundo tipo de sindicato, los bancos se comprometían a comprar firme los títulos que no hayan podido ser colocados en el mercado. En Londres, el sistema análogo era el llamado underwriting, que consistía en lo siguiente. Los bancos emisores, para asegurar el éxito de la emisión y no tomar sólos el riesgo de la emisión, entraban en contacto con otras instituciones o grandes inversionistas que, por una comisión de por medio (comisión de underwriting), tomaban el compromiso de adquirir una cierta cantidad de los títulos emitidos en el caso en el que el público no suscribiera la totalidad de la emisión.

\section{La preparación de la emisión}

Esta etapa dependía del tipo de sistema de emisión elegido. En el caso de los sindicatos, sus operaciones debían precisar desde un principio la cantidad de títulos que debían suscribir o garantizar, su tasa nominal y tasa de emisión para que los bancos tomaran la decisión de adhesión al sindicato. Era necesario ponerse de acuerdo sobre un cierto número de aspectos: la fecha de inicio y la duración del sindicato, los gastos que debían realizar y los beneficios esperados de la operación. Estos dependían de las comisiones según el sindicato del cual se tratara. El sindicato de adquisición en firme se beneficiaba de la diferencia entre el precio al cual el gobierno había cedido los títulos al sindicato y el precio de colocación en el mercado, deduciendo los gastos realizados. El sindicato de garantía exigía una comisión por título garantizado, una comisión de colocación por el número de títulos no colocados a repartir entre los participantes del sindicato además de una comisión de caja (comission de guichet). 


\section{La colocación de los títulos}

Existían tres maneras de colocar los títulos en el mercado: $a$ ) por suscripción pública, b) por introducción en la bolsa, y c) por medio de los bancos. La primera consistía en el anuncio de admisión de suscripciones públicas para una emisión en ciertas cajas designadas en el prospecto o en algún otro tipo de publicidad. En lo que refiere a la segunda, la introducción directa en los mercados bursátiles, la decisión importante por parte de los bancos emisores era el precio de introducción. Tchernoff explica que los límites de variación de este precio eran muy estrechos: en caso de que títulos del mismo género circularan en la bolsa, el precio de los nuevos títulos no podía ser superior. ${ }^{27}$ Además, la emisión de los títulos dependía en gran medida de las tendencias momentáneas. Es por esta razón que los bancos emisores recurrían frecuentemente a la realización de operaciones "ficticias" para aumentar el precio de los títulos: corredores de bolsa, underwriters o miembros de sindicatos compraban, en el momento de la publicación del prospecto aun antes, los títulos a emitir. Esta practica, considerada como un estimulante artificial de las emisiones, era considerada como manipulación de mercado (market rigging). Finalmente, siguiendo la tercera manera, los establecimientos bancarios podían poner a disposición de los emisores, sus sucursales o cajas, por una comisión (de colocación o de caja), o aun, si tal establecimiento formaba parte de un sindicato de garantía o de toma en firme, recomendaba los títulos a sus clientes.

El papel del intermediario financiero era por tanto fundamental para el éxito de cada emisión. Más aún, su papel era igualmente relevante para los inversionistas mismos, puesto que dichos bancos también actuaban como proveedores de consejo e información sobre las mejores posibilidades de inversión. Existían también los casos en que un país utilizara un solo banco para la emisión de deuda, con la posibilidad de sostener relaciones a largo plazo con sus ventajas y desventajas. En teoría financiera moderna, esto se denomina relación bancaria (relationship banking). ${ }^{28}$

Una relación de largo plazo entre un banco y un prestatario puede ser considerada como relación bancaria: esto facilita la supervisión continua sobre el prestatario y puede disminuir los problemas de información asimétrica. ${ }^{29}$ También permite la inclusión de cláusulas en los contratos de deuda específicos a cada caso (siendo por tanto más flexibles), la utilización de mecanismos más eficaces en caso de conflicto y el uso de colaterales

${ }^{27}$ Tchernoff, Syndicats, 1930.

${ }^{28} \mathrm{El}$ concepto de relationship banking utilizado en este contexto fue introducido por Flores, "Deuda", 2003; Flandreau, "Crises", 2003, y Flores, "Lorsque", 2004.

${ }^{29}$ Boot y Thakor, "Relationship", 2000. 
(que deben ser vigilados en el tiempo). Ongena y Smith mencionan igualmente la oportunidad para las empresas de construir una reputación de pagador puntual que se facilita con la existencia de la relación bancaria. ${ }^{30}$

Esta práctica bancaria también implica la provisión de cierta cantidad de servicios: inversión, por parte del banco, para obtener informaciones necesarias sobre el prestatario; evaluación de la rentabilidad de los proyectos a través de múltiples interacciones con el mismo prestatario. Para Greenbaum y Thakor la información obtenida puede ser reutilizada y mejorada por medio de la repetición de transacciones. ${ }^{31}$

Según Berger, existen tres condiciones necesarias para el desarrollo de una relación bancaria: $a$ ) el intermediario (banco) busca información más allá que la públicamente disponible, b) la cantidad de información aumenta con el número de interacciones, y $c$ ) la información permanece confidencial. ${ }^{32}$

Para Boot, el intercambio de la información resulta en una mejora en el sentido de Pareto. Por un lado, el prestatario puede tener más incentivos para revelar más información (ser más transparente; otro incentivo puede ser una demanda creciente de fondos). Por otro lado, el banco también tiene más incentivos a invertir en la producción de información. ${ }^{33}$

Existe un costo potencial para cada lado de la transacción. Para el banco, el costo se conoce como una "restricción suave de presupuesto" (soft budget constraint): la proximidad entre los dos agentes puede degenerar en una pérdida potencial de firmeza para hacer respetar los acuerdos. Para el prestatario, se puede presentar el problema de "retención" (hold-up): el banco genera un monopolio de la información en las transacciones, lo que le asegura la capacidad de cargar comisiones no competitivas en el futuro.

Una solución del prestatario ante el problema de retención consiste en recurrir a la competencia para hacer desaparecer las rentas de monopolio. Sin embargo, esto puede conducir a una "maldición del ganador" para los bancos competidores: como Rajan sostiene, los bancos que gozan de las rentas monopólicas pueden realizar ofertas competitivas para los buenos prestatarios y dejar a la competencia a los malos. ${ }^{34}$ Por el contrario, si los bancos están informados de manera más simétrica, las rentas monopólicas pueden desaparecer con la competencia. Sin embargo, el costo que permanece (para los bancos) es un control reducido sobre el comportamiento de inversión de los prestatarios.

Según Chan y Thakor, la correlación entre el grado de competencia de un mercado y la existencia de relación bancaria es negativa: los ban-

\footnotetext{
${ }^{30}$ Ongena y Smith, "What", 2000.

${ }^{31}$ Greenbaum y Takhor, Contemporary, 2007.

${ }^{32}$ Berger, "Big", 1999.

${ }^{33}$ Boot y Thakor, "Relationship", 2000.

${ }^{34}$ Rajan, "Insiders", 1992.
} 
cos anticipan una vida más corta de la relación con el prestatario. ${ }^{35}$ Estas expectativas disminuyen los incentivos para realizar las inversiones necesarias para el establecimiento de una relación bancaria. Al mismo tiempo, las economías de escala que surgen con la reutilización de la información disminuyen, lo que reduce el valor de esa información. Por otro lado, Petersen y Rajan analizan el impacto de la competencia sobre el precio de los empréstitos. ${ }^{36}$ Según estos autores, una competencia más importante restringe la capacidad de los bancos y de los prestatarios a compartir el excedente en el nivel inter temporal: los bancos cuentan con menos incentivos para "subsidiar" a los prestatarios en un primer periodo para aumentar las ganancias en el futuro.

\section{LA CRISIS BARING: UN FALLO DE MERCADO}

¿Cómo funcionaba la relación bancaria hace 100 años? El cuadro 1 muestra al conjunto de países cuyos gobiernos nacionales fueron prestatarios en Londres al menos en tres ocasiones entre 1870 y 1913 . Hemos incluido una columna con los datos sobre la estabilidad de la relación bancaria de cada país. El indicador que utilizamos es la proporción de emisiones cuyo intermediario financiero fue distinto al intermediario financiero de la emisión anterior (ratio de cambio). También incluimos datos sobre la prima de riesgo promedio y el número de defaults de cada país. ${ }^{37}$ Tal como argumentamos, la tendencia que observamos es que los países que cambian menos de intermediario financiero son aquellos países cuyos títulos presentan una menor prima de riesgo. Por otro lado, este cuadro se complementa con investigación previa respecto a la reputación misma de los intermediarios financieros, con Rothschild y Baring emitiendo títulos percibidos como menos riesgosos. ${ }^{38}$ De hecho, estos bancos eran los que más practicaban la relación bancaria. En periodos de dificultades financieras, los gobiernos buscaban emitir deuda a través de estos intermediarios financieros para garantizar el éxito de las emisiones, dada la reputación que gozaban estos bancos entre los inversionistas. Sabemos también que tanto Rothschild como Baring habían participado activamente en varios rescates

${ }^{35}$ Chan y Thakor, "Collateral”, 1987.

${ }^{36}$ Peterson y Rajan, "Effect”, 1995.

${ }^{37}$ La prima de riesgo la medimos como la diferencia del rendimiento de los títulos emitidos respecto al rendimiento de las consolas inglesas (UK consols, que eran activos financieros perpetuos de rentabilidad fija). Los empréstitos utilizados tienen un vencimiento de al menos diez años). Hemos dejado fuera los cross-listings (aquellos títulos que fueron introducidos en la bolsa de Londres sólo indirectamente).

${ }^{38}$ Véanse por ejemplo, Flandreau y Flores, "Bonds", 2009, o "Bondholders", 2011. 
Am. Lat. Hist. Econ., año 19, núm. 3, septiembre-diciembre, 2012, pp. 5-30

CUADRO 1. ROTACIÓN BANCARIA Y PRIMA DE RIESGO, 1870-1913

\begin{tabular}{ccccc}
\hline País & $\begin{array}{c}\text { Ratio de cambio } \\
\text { (en porcentajes) }\end{array}$ & $\begin{array}{c}\text { Prima de riesgo } \\
\text { (puntos base) }\end{array}$ & $\begin{array}{c}\text { Número de } \\
\text { emisiones }\end{array}$ & $\begin{array}{c}\text { Número de } \\
\text { defaults }\end{array}$
\end{tabular}

$\begin{array}{lrrrr}\text { Brasil } & 0.0 & 230 & 18 & 1 \\ \text { Dinamarca } & 0.0 & 80 & 4 & 0 \\ \text { Noruega } & 0.0 & 110 & 7 & 0 \\ \text { Japón } & 18.2 & 280 & 12 & 0 \\ \text { Cuba } & 25.0 & 220 & 5 & 0 \\ \text { Chile } & 33.3 & 200 & 18 & 1 \\ \text { Perú } & 33.3 & 320 & 3 & 1 \\ \text { China } & 36.0 & 570 & 26 & 0 \\ \text { Egipto } & 42.9 & 280 & 7 & 1 \\ \text { Grecia } & 43.8 & 250 & 16 & 2 \\ \text { Rusia } & 46.7 & 210 & 15 & 0 \\ \text { Bulgaria } & 50.0 & 290 & 3 & 0 \\ \text { Hungría } & 50.0 & 260 & 6 & 0 \\ \text { Suecia } & 50.0 & 130 & 8 & 0 \\ \text { Turquía } & 60.0 & 370 & 15 & 1 \\ \text { Uruguay } & 60.0 & 420 & 5 & 2 \\ \text { Argentina } & 66.7 & 280 & 24 & 1 \\ \text { Costa Rica } & 66.7 & 470 & 4 & 3 \\ \text { Francia } & 66.7 & 310 & 3 & 0 \\ \text { Portugal } & 66.7 & 290 & 6 & 1 \\ \text { Santo Domingo } & 66.7 & 480 & 4 & 4 \\ \text { México } & 100.0 & 380 & 7 & 1 \\ \text { Rumania } & 100.0 & 450 & 3 & 0 \\ \text { Tum } & & & & \end{array}$

Fuentes: elaboración propia con base en Suzuki, Japanese, 1994, complementado con The Times, 1870-1913, y The Investors Monthly Manual, 1870-1913; Klovland, "Pitfalls", 1994, para el cálculo de la prima de riesgo. Sobre los defaults, véase Suter, Debt, 1992.

financieros y negociaciones con países en default a lo largo del siglo XIX, incluyendo Turquía, México, Grecia o Brasil. La crisis Baring es un episodio relevante porque marcó un cambio abrupto en la estructura y jerarquía que había imperado en Londres hasta ese momento.

Tal como hemos sugerido antes, la crisis Baring también constituye una ilustración perfecta del cambio de paradigmas. También es interesante porque ha sido estudiada desde varias perspectivas en los últimos 100 años, acompañando la evolución de los debates en teoría económica del siglo XX. Ocupa un lugar privilegiado en la historiografía financiera ar- 
gentina e inglesa, poniendo en evidencia un caso en el que los problemas económicos de un país de la periferia podían a su vez afectar a los países del centro. Por primera vez, la situación requirió la intervención del banco de Inglaterra como prestamista de última instancia (como lo conocemos hoy en día), para evitar la caída del sistema bancario inglés. En fin, la crisis Baring constituye un caso particular que nos muestra cómo el mundo financiero del siglo XIX formaba sus percepciones, sus expectativas y cómo los inversionistas reaccionaban frente a eventos imprevistos.

La crisis Baring tuvo lugar en Argentina en el año de 1890. Se trató de una crisis "gemela", presentándose una crisis interna (bancaria) y externa (de moneda y de deuda). Una de las características de aquella crisis es su repercusión en el mundo financiero de la época. De hecho, el nombre de la crisis Baring pone en relieve la necesidad del rescate de la comunidad bancaria europea para evitar la caída del banco, uno de los principales del país y evitar así un pánico bancario. Baring había adquirido una importante proporción de su portafolio en títulos argentinos, que no pudo colocar en el mercado, y que corrían el riesgo de perder liquidez ante la moratoria inminente del gobierno de Argentina. Curiosamente, era la casa Baring el banco que llevaba una relación bancaria con aquel país a los ojos del mercado. Encontramos numerosas citas en la prensa de la época, así como trabajos más recientes que nos permiten corroborar que Baring tenía una relación particular con los gobiernos argentinos. Baring fue la primera en emitir títulos de deuda de la recientemente independizada Provincias Unidas de Río de la Plata en 1824 y quien más activa había sido para lograr un acuerdo con el gobierno varios años después del default. Además, Baring era la única casa bancaria en contar con un correspondiente permanente en Buenos Aires, Nicholas Bower, quien reportaba constantemente sobre proyectos en los que Baring tenía interés y sobre la situación económica general del país, particularmente el estado de las finanzas públicas. ${ }^{39} \mathrm{El}$ mercado financiero estaba al pendiente de esta situación. Reproducimos aquí una cita ilustrativa sobre la situación privilegiada de Baring respecto a la información sobre Argentina: "In Argentine the changes were again minute, and the bonds closed above the lowest prices of the day, notwithstanding the grave news published by Messrs. Baring Brothers this morning. They have received a telegram from Buenos Aires, dated yesterday, which was partially undecipherable, but it announced unmistakably that fighting had at length begun. ${ }^{\prime 0}$ Esta cita se refiere a uno de los frecuentes episodios en los que Buenos Aires, la provincia más rica de Argentina, se enfrentaba al resto de la confederación. En 1880, finalmente, se lograba una paz que

\footnotetext{
${ }^{39}$ Ferns, Britain, 1960.

40 "Money Market and City Intelligence", The Times, 24 de junio de 1880, p. 8.
} 
parecería duradera. Justamente, aquel año marcó el inicio de un boom de la inversión en Argentina (que llegó a su fin con la crisis Baring).

Baring dejó de ser el banco encargado de las emisiones argentinas a partir de 1880. Las condiciones favorables del país, su rápido crecimiento económico, su recuperación de la crisis de 1873 y sus sanas finanzas públicas parecen haber sido factores de atracción para que otros bancos propusieran sus servicios en términos más favorables que lo que Baring había propuesto en las décadas anteriores. ${ }^{41}$ En toda la década, los gobiernos argentinos trataron con diferentes bancos, dejando que competieran para disminuir los costos de los empréstitos.

La nueva competencia a partir de 1880 llegó en forma de sindicatos multinacionales de bancos provenientes principalmente de Francia en un principio, y a partir de 1885 de Alemania. Su oferta fue innovadora puesto que optaron muy frecuentemente por comprar los bonos "en firme", y esta modalidad les permitió hacer frente al dominio de la banca inglesa en la emisión de títulos como los argentinos, uruguayos o chilenos (en el caso argentino, Baring, así como Murrieta, el otro banco que había emitido bonos argentinos, habían actuado siempre individualmente). Por lo tanto, mientras la toma de riesgo por parte de los bancos emisores había sido nula o casi nula por la práctica de los banqueros ingleses que preferían las emisiones por medio de "venta a comisión", los bancos franceses promovieron con éxito la sindicalización en "firme". Esto permanecería así hasta $1913 .{ }^{42}$

Como hemos mencionado, existen numerosas teorías que intentan explicar la crisis argentina de 1890: distinguimos dos líneas que difieren entre sí en la causa primera del fenómeno. Por un lado, el enfoque monetarista, ${ }^{43}$ que insiste sobre las políticas monetarias expansionistas que llevó el gobierno argentino durante esa década. Por el otro, el enfoque de la balanza de pagos pone énfasis sobre los déficits comerciales cada vez más importantes que afectaron al país y que eran financiados mediante la entrada de flujos de capital externo. Cuando estos flujos dejaron de llegar a Argentina, la continuación del pago de la deuda fue insostenible, por lo que la crisis fue el resultado de un shock externo. Ambos tipos de teorías se centran en el aspecto macroeconómico de la crisis. No obstante, restan elementos no explicados. ¿Cómo fue posible que Argentina pudiera seguir endeudándose, cuando los banqueros sabían, incluso desde 1888, que tarde o temprano Argentina dejaría de pagar el servicio de la deuda? De hecho, hemos podido comprobar que por un lado la tasa de rendimiento de los

\footnotetext{
${ }^{41}$ Véanse Peters, Foreign, 1934; Jones, "European”, 1972; Marichal, Century, 1989, o Regalsky, "Exportaciones", 1987, y Mercados, 2001, sobre los factores que atrajeron las inversiones a Argentina.

${ }^{42}$ Un panorama general a largo plazo se encuentra en Flandreau et al., "End", 2010.

${ }^{43}$ Cortés, Dinero, 1989, y Paolera y Taylor, Straining, 2001.
} 
títulos argentinos para la segunda mitad de la década de 1880 se mantuvo sorprendentemente estable. Por el otro, el tipo de cambio peso argentinolibra esterlina experimentó una depreciación paulatina y continua. Esto es contrario a lo que la lógica financiera predice: una mayor depreciación de la moneda local tiene efectos negativos sobre el riesgo de impago sobre la deuda de un país, sobre todo si, como era el caso, la mayor parte de la deuda está denominada en moneda extranjera (libras esterlinas). Recordemos además que los ingresos gubernamentales estaban compuestos por moneda local.

Finalmente, quisiéramos remarcar, como algunos historiadores argumentan, que la crisis argentina era prevista incluso desde $1888 .^{44}$ Según Kindleberger ${ }^{45}$ los inversionistas siguieron comprando deuda argentina con la convicción de que Baring avalaba dichas emisiones, y que, en caso de problemas, Baring actuaría como prestamista en última instancia. No obstante, Baring había dejado de ser el único banco contratista del gobierno argentino, e incluso, actuaba como mera "caja" a cuenta de sindicatos continentales, recibiendo subscripciones a nuevas emisiones en el mercado de Londres o pagando los intereses sobre préstamos anteriores. Esta pérdida de negocio vino acompañada por el debilitamiento de sus fuentes de información de Argentina, principalmente por la relación cada vez más lejana con su agente en Buenos Aires.

Cuando la crisis estalló en Argentina en julio de 1890, muchos de los inversionistas voltearon su mirada a Baring. Uno de ellos, Foster \& Braithwaite, brokers en Londres, insistentemente pidió a Baring información sobre la "true financial condition of the Argentine Republic" ${ }^{46}$ Por medio de esta y otras citas semejantes podemos argumentar que, efectivamente, el mercado financiero europeo continuaba viendo a Baring como el banco que supervisaba la situación económica argentina y quien tendría que intervenir en caso de dificultades como había hecho siempre. Esta percepción ignoraba pues que la competencia en el mercado de deuda argentino había relegado a Baring a un segundo plano, y los incentivos para ejercer sus funciones de supervisor y prestamista de última instancia con Argentina habían disminuido.

Esta nueva situación contrastaba con la de otros países en América Latina. ${ }^{47}$ Brasil y Chile, muy activos en esos años, realizaron sus emisiones mediante unos pocos bancos, siendo Brasil el país que contaba con una

${ }^{44}$ Joslin, Century, 1963, o Eichengreen, “Baring”, 1999.

${ }^{45}$ Kindleberger, Panics, 1978.

${ }^{46}$ Archivos ING Baring, caja 4, 114.

${ }^{47}$ Sobre la relación entre los bancos y la competencia entre ellos véanse Marichal, "Banqueros", 1984; Juan H. Flores, "Lending Booms, Underwriting and Competition: The Baring Crisis Revisited", Working Papers in Economic History, Universidad Carlos III, enero de 2007, 
relación bancaria, puesto que el único emisor de sus títulos fue el banco de Rothschild. Al analizar los resultados del conjunto de emisiones de todos los países en la década de 1880, encontramos diferencias notables, que en un principio tendrían que estar relacionadas con los fundamentos económicos de cada país. Argentina aplicó, sobre todo a partir de 1887 (decreto de la ley de banca libre), políticas monetarias expansivas, contrario a las políticas restrictivas existentes tanto en Brasil como en Chile. Por otro lado, el endeudamiento de esos países no llegó a ser tan importante como el argentino. Chile era el país menos endeudado o con la mayor capacidad de repago. Argentina y Brasil tenían niveles similares de endeudamiento, pero el mayor aumento en este rubro tuvo lugar en Argentina.

En cuanto a los costos finales de las emisiones, encontramos lo siguiente. ${ }^{48}$ Por un lado, el gobierno argentino redujo considerablemente la prima de riesgo entre el primer empréstito y el último en 1889. Brasil también redujo su prima aunque no de la misma manera que Argentina, y también Chile (de hecho, este periodo se caracteriza por la baja general de tasas de interés, sobre todo de la tasa base que en aquel momento eran aquellas de los títulos de deuda ingleses). Podemos verificar que las tasas argentinas tienden a converger hacia las tasas brasileña y chilena. Esta tendencia también la observamos en las comisiones y márgenes de ganancia de los bancos por cada empréstito. El gobierno argentino comenzó pagando comisiones por el equivalente a $9 \%$ del valor nominal del primer empréstito, mientras que para 1889 estas comisiones se reducen a menos de $2 \%$. El gobierno de Chile, a lo largo de las emisiones deja poco margen de ganancia a los bancos, pagando incluso $1 \%$ en uno de sus empréstitos. Las comisiones al gobierno nacional de Brasil se mantuvieron constantes en alrededor de $2 \%$ a lo largo del periodo.

En resumen, podemos decir que en Argentina tuvimos la siguiente situación: competencia entre intermediarios financieros, deterioramiento de su situación macroeconómica, mayor posición de riesgo de los bancos, menor información fluida hacia el mercado y bancarrota de ambos, gobierno argentino e intermediario financiero principal en 1890. La quiebra y el consecuente rescate de Baring tuvieron efectos negativos sobre su parte de mercado en los empréstitos emitidos en Londres en el periodo posterior a 1890..$^{49}$ Argentina necesitó de dos reestructuraciones de deuda y no accedió al mercado de Londres de nuevo hasta principios del siglo xx. No obstante, la relación bancaria entre ambos continuó hasta 1913.

pp. 1-50, <http://www.uc3m.es/uc3m/dpto/HISEC/working_papers/working_papers_general. html>. [Consulta: 16 de abril de 2012], y Flores, "Competition", 2010.

${ }^{48}$ Un análisis detallado de las comisiones y costos de los empréstitos argentinos se encuentra en Regalsky, Mercados, 2001.

${ }^{49}$ Esto se demuestra en Flandreau et al., "End", 2010. 


\section{CONCLUSiOnes}

Aunque gran parte de la literatura en historia económica ha girado en torno al papel de los fundamentos económicos para determinar los costos de la deuda de los gobiernos prestatarios en Londres, trabajos más recientes ponen de relieve la importancia de la estructura de mercado y la reputación de los intermediarios financieros para comprender la relación entre los gobiernos y los mercados financieros internacionales. Fundamentos como la pertenencia al patrón oro como señal de buenas políticas económicas, muestran cada vez un menor poder explicativo de las primas de riesgo de los títulos de los gobiernos prestatarios en Londres, sobre todo a finales del siglo XIX. En cuanto a la crisis de 1890, Flandreau y Zumer han mostrado que mientras el grado de apertura económica era muy relevante antes de 1890, la variable que emerge en la década de 1890 es el del servicio de la deuda pública como proporción de los ingresos públicos. ${ }^{50}$ De hecho, este indicador mostraba en la década de 1880, que Argentina era ya un país problema varios años antes de la crisis. Aunque algunos economistas habían lanzado la voz de alarma, los bancos ignoraron este hecho y siguieron emitiendo deuda argentina por el incentivo de los beneficios, siendo acusados después de la crisis de tener conflictos de interés y de no haber advertido a los inversionistas sobre los riesgos de las nuevas emisiones.

Esta situación de mercado llevó a un resultado semejante al de las crisis contemporáneas. Al igual que en el periodo actual, los supervisores de las buenas políticas económicas eran los intermediarios financieros, cuyo último objetivo era el beneficio de sus operaciones inmediatas, por lo que no siempre tenían incentivos para realizar de la mejor manera el seguimiento a la situación de sus prestatarios. Por lo mismo, el mercado comenzó a producir su propia información al margen de los bancos emisores. Como bien muestra Flandreau, es evidente que la necesidad de contar con información precisa y confiable después de la crisis de 1890 llevó a la creación del Servicio de Estudios Financieros de Crédit Lyonnais, movimiento imitado en otras partes de Europa y Estados Unidos ${ }^{51}$ con la emergencia de Moody's en la década de 1920 como principal agencia de rating. Las publicaciones especializadas tuvieron un importante auge en la década de 1890, así como la información contenida en reportes anuales como el de la Corporation of Foreign Bondholders. Desde entonces, los parámetros utilizados para calificar la situación de un país también han cambiado, incluso en el presente, nos sorprenderíamos al conocer cuáles eran los parámetros en la década de 1920.

\footnotetext{
${ }^{50}$ Flandreau y Zumer, Making, 2004.

${ }^{51}$ Flandreau, "Cavaet”, 1998.
} 
La estructura de la información es importante bajo cualquier punto de vista e independientemente del periodo analizado. Pero también lo es la existencia de incentivos de mercado que eviten la emergencia de conflictos de interés o, en su defecto, la introducción de un ente regulador cuyo incentivo no pueda ser influido por variables diferentes a las económicas, y cuyo objetivo preciso y final sea la estabilidad de la arquitectura financiera internacional.

\section{FUENTES CONSULTADAS}

\section{Archivos}

AIB Archivos ING Baring, Londres, Inglaterra.

\section{Hemerografia}

South American Journal, Londres, Reino Unido.

The Economist, Londres, Reino Unido.

The Times, Londres, Reino Unido.

The Investors Monthly Manual, Londres, Reino Unido.

\section{Bibliografía}

Accominotti, Olivier, Marc Flandreau y Riad Rezzik, "The Spread of Empire:

Clio and the Measurement of Colonial Borrowing Costs", The Economic History Review, University of Cambridge/National Bureau of Economic Research, vol. 64, núm. 2, 2011, Cambridge, pp. 385-407.

Ahvenainen, Jorma, The European Cable Companies in South America before the First World War, Helsinki, Academia Scientiarum Fennica, 2004, 427 pp.

Akerlof, George A., "The Market for Lemons: Quality Uncertainty and the Market Mechanism", Quarterly Journal of Economics, Oxford University Press, vol. 84, núm. 3, 1970, Oxford, pp. 488-500.

Berger, Allen N., "The 'Big Picture' about Relationship-Based Finance” en Jackson L. Blanton, Alicia Williams y Sherrie L. W. Rhine (eds.), Business Access to Capital and Credit, Federal Reserve System Research Conference, Arlington, 1999, pp. 390-400.

BoOt, ARnoud W. A., "Relationship Banking: What Do We Know?", Journal of Financial Intermediation, Elsevier, vol. 9, núm. 1, 2000, Illinois, pp. 7-25. 
Boot, Arnoud W. A. y Anjan V. Thakor, "Moral Hazard and Secured Lending in an Infinitely Repeated Credit Market Game”, International Economic Review, University of Pennsylvania, vol. 35, núm. 4, 1994, Philadelphia, pp. 899-920. “Can Relationship Banking Survive Competition?", The Journal of Finance, The American Finance Association, vol. 55, núm. 2, abril de 2000, Berkeley, pp. 679-713.

Bon, Gustave Le, Psychologie des foules, París, F. Alcan, 1895.

Bordo, Michael David, Barry Eichengreen y Douglas A. Irwin, "Is Globalization Today Really Different than Globalization a Hundred Years Ago?” en SusAN Collins y Robert Lawrence (eds.), Brookings Trade Policy Forum, Washington, Brookings Institution, 1999, pp. 1-50.

Cairncross, Alec K., Home and Foreign Investment, 1870-1913, Studies in Capital Accumulation, Cambridge, University Press, 1953, 251 pp.

Calvo, Guillermo A., "Capital Flows and Capital-Market Crises: The Simple Economics of Sudden Stops", Journal of Applied Economics, Universidad del Centro de Estudios Macroeconómicos de Argentina, vol. 1, núm. 1, 1998, Buenos Aires, pp. 35-54.

"Contagion in Emerging Markets: when Wall Street is a carrier", The International Economic Association Congress, vol. 3, Buenos Aires, 2002.

Chan, Yuk-Shee y Anjan V. Thakor, "Collateral and Competitive Equilibria with Moral Hazard and Private Information", The Journal of Finance, The American Finance Association, vol. 42, núm. 2, junio de 1987, Berkeley, pp. 345-363.

Chemmanur, Thomas J. y Paolo Fulghieri, "Reputation, Renegotiation, and the Choice between Bank Loans and Publicly Traded Debt", Review of Financial Studies, Society for Financial Studies, Oxford University Press, vol. 7, núm. 3, 1994, Oxford, pp. 475-506.

Clemens, Chael A. y Jeffrey G. Williamson, "Wealth Bias in the First Global Capital Market Boom, 1870-1913”, The Economic Journal, Royal Economic Society, vol. 114, núm. 495, abril de 2004, Massachusetts, pp. 304-337.

Cortés Conde, R., Dinero, deuda y crisis: evolución fiscal y monetaria en la Argentina, $1862-$ 1890, Buenos Aires, Sudamericana, 1989.

DeLong, James Bradford, "Economics in Crisis", The Economists' Voice, The Berkeley Electronic Press, vol. 8, núm. 2, 2011, Berkeley, <http://www.bepress.com/ev/ vol8/iss2/art2>. [Consulta: 16 de abril de 2012.]

Dornbusch, Rudiger, Yung Chul Park y Stijn Claessens, "Contagion Understanding How it Spreads", The World Bank Research Observer, The International Bank for Reconstruction and Development, Oxford University Press, vol. 15, núm. 2, 2000, Oxford, pp. 177-197.

Edelstein, Michael, "Foreign Investment and Accumulation, 1860-1914" en RoderICK Floud y Deirdre N. MCCloskey (eds.), The Economic History of Britain since 1700, vol. 2: 1860-1939, Cambridge, Cambridge University Press, 2a. ed., 1994, pp. 173-196. 
Eichengreen, Barry, "The Baring Crisis in a Mexican Mirror", International Political Science Reviere, International Political Science Association, vol. 20, núm. 3, 1999, Londres, pp. 249-270.

Capital Flows and Crises, Cambridge Massachusetts, Massachusetts Institute of Technology Press, 2003, 377 pp.

y Michael D. Bordo, "Crises Now and Then: What Lessons from the Last Era of Financial Globalization?”, The National Bureau of Economic Research, enero de 2002, Massachusetts, pp. 1-56 (Working Paper Series núm. 8716).

Fenn, Charles, Fenn's Compendium of the English and Foreign Funds, Debts and Revenues of all Nations..., Londres, Effingham Wilson, 1883.

Ferns, Henri Stanley, Britain and Argentina in the Nineteenth Century, Oxford, Clarendon Press, 1960, 517 pp.

"The Baring Crisis Revisited", Journal of Latin American Studies, Cambrigde University Press, vol. 24, núm. 2, 1992, Cambrigde, pp. 241-73.

Ferguson, Niall y Moritz Schularick, "The Empire Effect: The Determinants of Country Risk in the First Age of Globalization, 1880-1913”, Journal of Economic History, University of Cambridge/National Bureau of Economic Research, vol. 66, núm. 2, 2006, Cambrigde, pp. 283-312.

Ferri, Giovanni, Li-Gang Liu y Joseph E. Stiglitz, "The Procyclical Role of Rating Agencies: Evidence from the East Asian Crisis", Economic Notes, Review of Banking, Finance and Monetary Economics, Universidad de Siena, vol. 28, núm. 3, 1999, Italia, pp. 335-355.

Flandreau, Marc, "Cavaet Emptor: Coping with Sovereign Risk without the Multilaterals”, Discussion Paper, Fondation Nationale des Sciences Politiques/Institut d'Etudes Politiques de Paris/Centre for Economic Policy Research, núm. 2004, 1998, París, 41 pp.

"Crises and Punishment: Moral Hazard and the Pre-1914 International Financial Architecture" en Marc Flandreau (ed.), Money Doctors: The History, Theory and Policy of International Financial Advising, 1850-2000, París, Routledge, 2003, pp. 13-48.

"Home Biases, Nineteenth Century Style", Journal of the European Economic Association, Universidad de Zurich, vol. 4, núm. 2-3, 2006, Zurich, pp. 634-643.

et al., "The End of Gatekeeping: Underwriters and the Quality of Sovereign Bond Markets, 1815-2007" en Lucrezia Reichlin y Kenneth D. West (eds.), National Bureau of Economic Research, International Seminar on Macroeconomics 2009, Chicago, Chicago University Press, 2010, pp. 53-92.

Flandreau, Marc y Fréderic Zumer, The Making of Global Finance, 1880-1913, París, Organización para la Cooperación y Desarrollo Económico, 2004, 144 pp.

Flandreau, Marc y Juan H. Flores, "Bonds and Brands. Lessons from the 1820s", Journal of Economic History, University of Cambridge/National Bureau of Economic Research, vol. 69, núm. 3, 2009, Cambridge, pp. 646-684. 
"Bondholders vs. Bond-sellers? Investment Banks and Conditionality Lending in the London Market for Foreign Government Debt, 1815-1913", European Historical Economics Society, enero de 2011, pp. 1-44 (EHES Working Paper Series núm. 2), <http://ehes.org/EHES_No2.pdf>. [Consulta: 16 de abril de 2012.]

Flores, Juan H., "Deuda Pública y el concepto de Relationship Banking a finales del siglo XIX: un caso histórico de Delegated Monitoring?”, artículo presentado para la V Reunión de Economía Mundial, Universidad de Sevilla, 2003.

"Lorsque le Leader suit la foule. La crise Baring dans une perspective microéconomique", tesis de doctorado, Francia, Instituto de Estudios Políticos de París, 2004.

"Competition in the Underwriting Markets of Sovereign Debt: The Baring Crisis Revisited", Law and Contemporary Problems Quarterly, Duke University School of Law, vol. 73, núm. 4, 2010, Durham, pp. 129-150.

Gaytan, Alejandro y Christian A. Johnson, "A Review of the Literature on Early Warning Systems for Banking Crises", Santiago de Chile, Banco Central de Chile, 2002, pp. 1-45 (Documento de trabajo, núm. 183).

Greenbaum, Stuart I. y Anjan V. Thakor, Contemporary Financial Intermediation, Nueva York, Dryden Press, 2007, 643 pp.

Jones, Charles A., "European Bankers and Argentine, 1880-1890”, Cambridge, Centre of Latin American Studies-University of Cambridge, 1972, pp. 1-13 (Working Papers, Business Imperialism Series, núm. 3).

Joslin, David, A Century of Banking in Latin America; to Commemorate the Centenary in 1962 of the Bank of London E South America Limited, Nueva York, Oxford University Press, 1963, 307 pp.

Kaminsky, Graciela Laura y Carmen M. Reinhart, "The Twin Crises: The Causes of Banking and Balance-of-Payments Problems", American Economic Review, American Economic Association, Vanderbilt University, vol. 89, núm 3, 1999, Nashville, Tennessee, pp. 473-500.

Kindleberger, Charles H. Manias, Panics, and Crashes: A History of Financial Crises, Nueva York, John Wiley \& Sons, 4a. ed., 1978.

Klovland, Jan TORE, "Pitfalls in the Estimation of the Yield on British Consols, 18501914", Journal of Economic History, University of Cambridge/National Bureau of Economic Research, vol. 54, núm. 1, 1994, Cambridge, pp. 164-187.

Krugman, Paul R., The Return of Depression Economics, Nueva York, W. W. Norton \& Company, 1999, 176 pp.

Kunn, Thomas S., The Structure of Scientific Revolutions, Chicago, The University of Chicago Press, 2a ed., 1970, 197 pp.

Landes, David S., "Why are We So Rich and They So Poor?", American Economic Reviere, Papers and Proceedings of the Hundred and Second Annual Meeting of the American Economic Association, Vanderbilt University, vol. 80, núm. 2, 1990, Nashville, Tennessee, pp. 1-13. 
Ludlow, Leonor y Carlos Marichal Salinas (coords.), Un siglo de deuda pública en México, México, Instituto de Investigaciones Dr. José María Luis Mora/El Colegio de Michoacán/El Colegio de México/Universidad Nacional Autónoma de México, 1998, 269 pp.

Lummer, Scott L. y John J. McConnel, "Further Evidence on the Bank Lending Process and the Market Response to Bank Loan Agreements", The Journal of Financial Economics, Cambrigde University Press/Economic History Association, vol. 25, núm. 1, 1989, Cambrigde, pp. 99-122.

Marichal Salinas, Carlos, "Los banqueros europeos y los empréstitos argentinos: rivalidad y colaboración, 1880-1890”, Revista de Historia Económica, Instituto Figuerola de Historia y Ciencias Sociales-Universidad Carlos III de Madrid, vol. 2, núm. 1, 1984, Madrid, pp. 47-82.

A Century of Debt Crises in Latin America: From Independence to the Great Depression, 1820-1930, Princeton, Nueva Jersey, Princeton University Press, 1989, 304 pp.

, Nueva historia de las grandes crisis financieras. Una perspectiva global, 18732008, México, Debate, 2010, 420 pp.

Mauro, Paolo, Nathan Sussman y Yishay Yafeh, Emerging Markets and Financial Globalization. Sovereign Bond Spreads in 1870-1913 and Today, Oxford, Oxford University Press, 2006, $193 \mathrm{pp}$.

Mosley, Layna, Global Capital and National Governments, Cambridge, Cambridge University Press, 2003, 337 pp.

Mulhall, George Michael, Mulhall's Dictionary of Estadistics, Montana, Kessinger Publishing, 2008, 544 pp. (edición de la de 1886).

NuRSKe, RAGNAR, "International Investment Today in the Light of 19th Experience", The Economic Journal, Royal Economic Society/University of Cambridge, vol. 64, núm. 256, 1954, Cambrigde, pp. 744-58.

Obstfeld, Maurice y Alan M. Taylor, "Globalization and Capital Markets" en Michael D. Bordo, Alan M. Taylor y Jeffrey G. Williamson, Globalization in Historical Perspective, Chicago, The National Bureau of Economics Research, University of Chicago Press, 2003, pp. 121-188.

Ongena, Steven y David C. Smith, "What Determines the Number of Bank Relationships? Cross-Country Evidence”, Journal of Financial Intermediation, Elsevier, vol. 9, núm. 1, 2000, Illinois, pp. 26-56.

Paolera, Gerardo Della y Alan M. Taylor, Straining at the Anchor: the Argentine Currency Board and the Search for Macroeconomic Stability, 1880-1935, Chicago, University of Chicago Press, 2001.

Peters, Harold Edwin, The Foreign Debt of the Argentine Republic, Baltimore, The John Hopkins Press, 1934, 186 pp.

Peterson, Mitchell A. y Raghuram G. Rajan, "The Effect of Credit Market Competition on Lending Relationships", Quarterly Journal of Economics, National Bureau 
of Economics Research, Oxford University Press, vol. 110, núm. 2, 1995, Oxford, pp. 406-443.

Pike, Robert M. y Dwayne Roy Winseck, Communication and Empire. Media, Markets, and Globalization, 1860-1930, Durham, Carolina del Norte, Duke University Press, 2007, 406 pp.

Quinn, Dennis P., "Capital Account Liberalization and Growth, 1890-1999: A Synoptic View”, International Journal of Finance and Economics, John Wiley and Sons, vol. 8, núm. 3, 2003, New Jersey, pp. 189-204.

Radelet, Steven y Jeffrey D. Sachs, "The East Asian Financial Crisis: Diagnosis, Remedies, Prospects", Brookings Papers on Economic Activity, Brookings Institution, núm. 1, vol. 29, 1998, Washington, pp. 1-90.

Rajan, Raghuram G., "Insiders and Outsiders: The Choice between Informed and Arm's-Length Debt", The Journal of Finance, The American Finance Association, vol. 47, núm. 4, septiembre de 1992, Berkeley, pp. 1367-1400.

REgAlSKY, Andrés M., "Exportaciones de capital hacia los países nuevos: los bancos franceses y las finanzas públicas argentinas, 1881-1887", Revista de Historia Económica-Journal of Iberian and Latin American Economic History, Universidad de La Rioja, vol. 5, núm. 1, 1987, La Rioja, pp. 73-97.

Mercados, inversiones y elites. Las inversiones francesas en la Argentina, 18801914, Buenos Aires, Universidad Nacional Tres de Febrero/Editorial de la Universidad Nacional Tres de Febrero, 2001.

Rodrik, Dani y Arvind Subramanian, "Why Did Financial Globalization Disappoint?", International Monetary Fund. Staff Papers, Palgrave Macmillan Journals/Fondo Monetario Internacional, vol. 56, núm. 1, marzo de 2009, pp. 112-138, <http:// www.iie.com/publications/papers/subramanian0308.pdf $>$. [Consulta: 16 de abril de 2012.]

Stone, Inving, The Global Capital Export from Great Britain, 1865-1914, Nueva York, St. Martin Press, 1999.

Suter, Christian, Debt Cycles in the World-Economy. Foreign Loans, Financial Crises and Debt Settlements, 1820-1990, Boulder, Westview Press, 1992.

Suzuki, Toshio, Japanese Government Loan Issues on the London Capital Market, 18701913, Londres, Athlone Press, 1994.

TCHERnOFF, IOUDA, Les syndicats financiers: syndicats d'émission et de placement, syndicats de blocage, syndicats de résistance, syndicats de bourse, investment trust et holding suivi de formules d'application, París, Librairie du recueil Sirey, 1930.

Thakor, Anjan V., "Capital Requirements, Monetary Policy and Aggregate Bank Lending: Theory and Empirical Evidence", Journal of Finance, Blackwell Plublishing/ American Finance Association, vol. 51, núm. 1, 1996, pp. 279-324. 